Characteristic length of phonon transport within periodic nanoporous thin films and two-dimensional materials

Qing Hao, Yue Xiao, and Hongbo Zhao

Citation: J. Appl. Phys. 120, 065101 (2016); doi: 10.1063/1.4959984

View online: http://dx.doi.org/10.1063/1.4959984

View Table of Contents: http://aip.scitation.org/toc/jap/120/6

Published by the American Institute of Physics 


\title{
Characteristic length of phonon transport within periodic nanoporous thin films and two-dimensional materials
}

\author{
Qing Hao, ${ }^{\text {a) }}$ Yue Xiao, and Hongbo Zhao \\ Department of Aerospace and Mechanical Engineering, University of Arizona, Tucson, \\ Arizona 85721-0119, USA
}

(Received 28 April 2016; accepted 17 July 2016; published online 8 August 2016)

\begin{abstract}
In the past two decades, phonon transport within nanoporous thin films has attracted enormous attention for their potential applications in thermoelectrics and thermal insulation. Various computational studies have been carried out to explain the thermal conductivity reduction within these thin films. Considering classical phonon size effects, the lattice thermal conductivity can be predicted assuming diffusive pore-edge scattering of phonons and bulk phonon mean free paths. Following this, detailed phonon transport can be simulated for a given porous structure to find the lattice thermal conductivity [Hao et al., J. Appl. Phys. 106, 114321 (2009)]. However, such simulations are intrinsically complicated and cannot be used for the data analysis of general samples. In this work, the characteristic length $\Lambda_{\text {Pore }}$ of periodic nanoporous thin films is extracted by comparing the predictions of phonon Monte Carlo simulations and the kinetic relationship using bulk phonon mean free paths modified by $\Lambda_{\text {Pore }}$. Under strong ballistic phonon transport, $\Lambda_{\text {Pore }}$ is also extracted by the Monte Carlo ray-tracing method for graphene with periodic nanopores. The presented model can be widely used to analyze the measured thermal conductivities of such nanoporous structures. Published by AIP Publishing. [http://dx.doi.org/10.1063/1.4959984]
\end{abstract}

\section{INTRODUCTION}

In recent years, nanoporous thin films have been widely studied for their potential applications in thermoelectrics, ${ }^{1-17}$ gas sensors, ${ }^{18}$ and thermal management. ${ }^{19-21}$ For thermoelectrics, the effectiveness of a material is evaluated by its thermoelectric figure of merit (ZT), defined as $\mathrm{ZT}=S^{2} \sigma T / k$, where $S, \sigma, k$, and $T$ represent Seebeck coefficient, electrical conductivity, thermal conductivity, and absolute temperature, respectively. ${ }^{22}$ In semiconducting thermoelectric materials, $k$ can be further split into the lattice contribution $k_{L}$ and electronic contribution $k_{E}$. By introducing periodic nanopores, the lattice thermal conductivity $k_{L}$ can be largely suppressed in these samples but bulk-like $S^{2} \sigma$ can still be conserved, leading to enhanced thermoelectric performance. ${ }^{4,8}$ A room-temperature $\mathrm{ZT} \sim 0.4$ has been reported for nanoporous Si films with pitch of $55 \mathrm{~nm}$ and $35 \%$ porosity, in which the measured $k$ values are two orders of magnitude lower than that of the bulk $\mathrm{Si}^{8}$ Besides thin films, periodic nanopores or even atomic pores can also be patterned on two-dimensional (2D) materials such as graphene to improve its ZT, which is also called graphene antidot lattices (GALs) in the literature. ${ }^{23}$ With down to $\sim 1 \mathrm{~nm}$ periods, an electronic band gap can be possibly opened in semimetal graphene to dramatically increase its low $S$ and thus $S^{2} \sigma$. Combined with the $k_{L}$ reduction, $\mathrm{ZT} \sim 1.0$ at $300 \mathrm{~K}$ has been predicted for GALs ${ }^{24}$ though experimental validation is still lacking.

Theoretically, numerous efforts have been dedicated to explaining the observed $k_{L}$ reduction of nanoporous $\mathrm{Si}$ thin films, with pores drilled either with a focused ion beam or

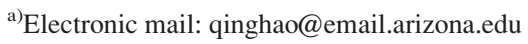

reactive ion etching (RIE). Considering particle-like phonons, frequency-dependent phonon Monte Carlo (MC) simulations were carried out to compute $k_{L}$, in which diffusive phonon scattering by rough pore edges and bulk phonon mean free paths (MFPs) were assumed. ${ }^{10,11}$ However, it was found that $k_{L}$ was often lower than predictions by phonon MC simulations for fine porous structures. This divergence is attributed to two new mechanisms, i.e., phonon coherence and pore-edge defects. ${ }^{25}$ First, periodic nanopores may lead to modified phonon dispersion due to coherent interference of lattice vibrations as waves, which reduces the phonon group velocities and therefore $k_{L}{ }^{3,4,14,15}$ Considering the wave nature of phonons, this so-called "phononic effect" leads to reduced $k_{L}$ in a superlattice with periodic interfaces. $^{26-28}$ However, the impact of phononic effects is negligible in the measured nanoporous films with a period that is much larger than the dominant phonon wavelength $\lambda(\sim 1 \mathrm{~nm}$ for Si at $300 \mathrm{~K}$ (Ref. 22)). In comparable superlattices with well-defined periodic interfaces, phonon coherence is important only for sub- $10 \mathrm{~nm}$ periods at $300 \mathrm{~K}$, whereas diffusive phonon scattering by interfaces is dominant for larger periods. ${ }^{29,30}$ Consistently, more recent analysis shows that diffusive pore-edge phonon scattering is sufficient to explain the data of nanoporous films with feature sizes larger than $100 \mathrm{~nm}$ at $300 \mathrm{~K}^{31}$ As the second mechanism, pore-edge amorphization and oxidation, as found in real Si thin films, ${ }^{8}$ may largely affect $k_{L}$ for fine nanoporous structures. ${ }^{1}$ Such amorphous and surface oxidation layers usually have a few $\mathrm{nm}$ thickness. In practice, these pore-edge defects will slightly expand the effective pore diameter in phonon $\mathrm{MC}$ simulations. $^{32}$

Assuming diffusive phonon scattering by pore edges, $k_{L}$ can be predicted using the kinetic relationship by modifying 
the bulk phonon MFPs with the characteristic length $\Lambda_{\text {Pore }}$ of the nanoporous structure. Similar treatment has been taken for nanowires, in which the nanowire diameter is used to modify the bulk phonon MFPs. ${ }^{33}$ Along this line, the phonon MC ray tracing (MCRT) method has been employed to extract either $\Lambda_{\text {Pore }}$ for nanoporous bulk materials ${ }^{34}$ or the modified phonon MFPs for porous films. ${ }^{13}$ However, the extracted $\Lambda_{\text {Pore }}$ often leads to divergence between $k_{L}$ predicted by the kinetic relationship and phonon MC simulations considering frequency-dependent phonon MFPs. On the other hand, extracting the modified phonon MFPs for a given structure dimension is very time-consuming when frequency-dependent phonon MFPs are considered.

Instead of modifying phonon MFPs, $k_{L}$ can be directly provided by phonon MC simulations that track the detailed phonon movement and scattering processes. ${ }^{10,11,31,32,35}$ Other $k_{L}$ predictions are based on the numerical ${ }^{6,7,36,37}$ and analytical ${ }^{38,39}$ solutions of the phonon Boltzmann transport equation (BTE). However, these studies are still complicated and cannot be used for the thermal analysis of general samples with varied porous structures.

In this work, nanoporous films with sub- $100 \mathrm{~nm}$ to submicron feature sizes are modeled for the in-plane $k_{L}$. Specular phonon reflection by the top and bottom surfaces of a film is assumed so that the film thickness does not have any influence on $k_{L}$. In real thin films, it should be noted that certain film-boundary roughness can introduce partially diffusive phonon scattering and thus lower $k_{L}$ at reduced film thickness. ${ }^{40-43}$ When the film thickness is comparable or even smaller than the size of the porous structure, more accurate modeling may further consider the film-boundary scattering of phonons. ${ }^{13}$ Assuming smooth film boundaries and rough pore edges, an effective $\Lambda_{\text {Pore }}$ is extracted by directly comparing the $k_{L}$ predicted by phonon MC simulations and the kinetic relationship. Classical phonon size effects are considered because phonon coherence can become critical only for sub- $10 \mathrm{~nm}$ periods at $300 \mathrm{~K}$ or higher temperatures. The study is further extended to atomic-thick materials with periodic pores and dominant ballistic phonon transport. In this case, the cross-plane-component of the phonon group

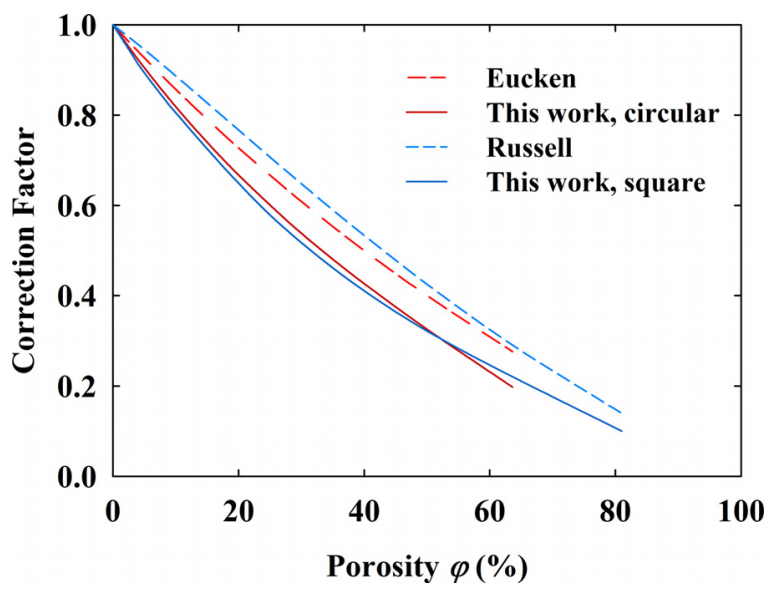

FIG. 1. New correction factors for GALs and nanoporous thin films with through-film pores, in comparison with the Russell model and Eucken model. velocity is fixed at zero. In general, the extracted $\Lambda_{\text {Pore }}$ can be widely used for the thermal analysis of nanoporous thin films. The simple analysis based on the kinetic relationship can replace the time-consuming phonon MC simulations considering frequency-dependent phonon MFPs.

\section{CHARACTERISTIC LENGTH AND ITS VALIDATION}

\section{A. Analytical model for $k_{L}$ and correction factors}

The lattice thermal conductivity $k_{L}$ of a nanoporous material is computed based on the kinetic relationship

$$
k_{L}=\frac{1}{3} F(\varphi) \sum_{i=1}^{3} \int_{0}^{\omega_{\max , i}} c_{i}(\omega) v_{g, i}(\omega) \Lambda_{i}(\omega) d \omega
$$

where $F(\varphi)$ is the correction factor for porosity $\varphi, \omega$ is the phonon angular frequency, the subscript $i$ indicates the phonon branch, $c_{i}(\omega)$ is the spectral volumetric phonon specific heat, $v_{g, i}(\omega)$ is the phonon group velocity, and $\Lambda_{i}(\omega)$ is the modified phonon MFP for branch $i$ and angular frequency $\omega$. Only three acoustic branches are considered here because the optical phonon contribution is negligible due to their small group velocities. Based on the Matthiessen's rule, $\Lambda_{i}(\omega)$ is modified from the bulk phonon MFP $\Lambda_{B u l k, i}(\omega)$ as

$$
\frac{1}{\Lambda_{i}(\omega)}=\frac{1}{\Lambda_{\text {Bulk,i },}(\omega)}+\frac{1}{\Lambda_{\text {Pore }}}
$$

in which $\Lambda_{\text {Pore }}$ is the characteristic length of the porous structure. The focus of this study is to better predict $\Lambda_{\text {Pore }}$ for general two-dimensional periodic porous structures.

For a bulk material with cubically aligned spherical pores, the correction factor $F(\varphi)$ is proposed by Eucken, given as ${ }^{44,45}$

$$
F(\varphi)=\frac{1-\varphi}{1+\varphi / 2}
$$

For a bulk material with cubic pores, $F(\varphi)$ is changed to the Russell factor ${ }^{46}$

$$
F(\varphi)=\frac{1-\varphi^{2 / 3}}{1+\varphi-\varphi^{2 / 3}} .
$$

Despite their usage in porous thin films, ${ }^{2,8,13}$ these factors were only derived for three-dimensional (3D) bulk porous materials. For porous thin films, Fourier analysis was thus carried out to compute $F(\varphi)$ for different pore configurations with $\varphi<30 \%{ }^{7}$ Using the Analysis System (ANSYS) package, here $F(\varphi)$ is computed for macroscale 2D porous structures with a wider $\varphi$ range (Fig. 1). At very high porosities, the accurate $F(\varphi)$ may diverge from the Eucken or Russell factors by up to $33 \%$.

\section{B. $\Lambda_{\text {Pore }}$ prediction for ballistic phonon transport within periodic porous thin films}

One way to extract $\Lambda_{\text {Pore }}$ for a given porous structure is by the MCRT method. In this method, a single period is normally selected as the computational domain, with the pore in 
its center. Phonons randomly distributed within the domain will move with their own velocities. For simplicity, the same velocity $v_{g}$ is assigned to all phonons. Phonons hitting the left or right boundaries of the domain will re-enter the domain from the opposite boundary by shifting the phonon location with the domain length. Phonons incident on other domain boundaries will be specularly reflected, whereas the pore edge will diffusively scatter the phonons. The above boundary conditions are consistent with phonon MC simulations for thin films, in which heat flows from the left boundary to the right boundary. No phonon-phonon scattering is considered here. The pore edge scattering will reset the traveling direction of phonons, the detailed treatment for circular pores is given in Appendix A and the treatment for rectangular pores has been provided in earlier studies. ${ }^{10,11}$

Within a certain time step $\Delta t$, the percentage of phonons not scattered by the pore edge $\left(P_{\text {Unscatt }}\right)$ will be used to compute $\Lambda_{\text {Pore }}{ }^{47}$

$$
\Lambda_{\text {Pore }}=\frac{v_{g} \Delta t}{\ln \left(\frac{1}{P_{\text {Unscatt }}}\right)}
$$

For better accuracy, several million phonons are typically used in the MCRT method, and more than 5000 time steps are carried out to obtain an average $P_{\text {Unscatt }}$. The time step $\Delta t$ is reduced to roughly $\Lambda_{\text {Pore }} / 10 v_{g}$, and no influence on $\Lambda_{\text {Pore }}$ is found. As a geometry-determined value, the dimensionless $\Lambda_{\text {Pore }}^{*}=\Lambda_{\text {Pore }} / p$ changes within $1 \%$ in all simulated cases with the same porosity and pore shape, regardless of the period $p$ and film thickness.

Without MC simulations, $\Lambda_{\text {Pore }}$ can also be estimated by the mean beam length (MBL) for an enclosure, which is widely used in radiation. Under the optically thin limit, the MBL is given as ${ }^{48}$

$$
M B L=\frac{4 V}{S}
$$

where the solid-region volume $V$ and pore surface area $S$ can be evaluated for a given porous structure. Here, MBL assumes ballistic phonon transport within a 3D enclosure, while phonons in the MCRT simulation start from random locations within the nanoporous material. Despite the difference in their definitions, the MCRT method and MBL give identical $\Lambda_{\text {Pore }}^{*}$ for thin films with either square or circular pores, as shown in Fig. 2. In addition, this $\Lambda_{\text {Pore }}^{*}$ is independent of the period $p$ and film thickness.

It should also be noted that $\Lambda_{\text {Pore }}$ can be estimated following the derivation of the gas molecule MFPs based on the diameter and number density of these molecules. ${ }^{22}$ In previous studies, the same process is used to derive the phonon MFPs due to nanoparticle scattering within a particle-in-a-host nanocomposite. ${ }^{49}$ For $2 \mathrm{D}$ porous structures, similar process gives

$$
\Lambda_{\text {Pore }}=\frac{p^{2}}{d}=\frac{\pi d}{4 \varphi},
$$

in which $d$ is the diameter of a circular pore. However, this characteristic length becomes invalid when $\varphi$ is close to 1 . In

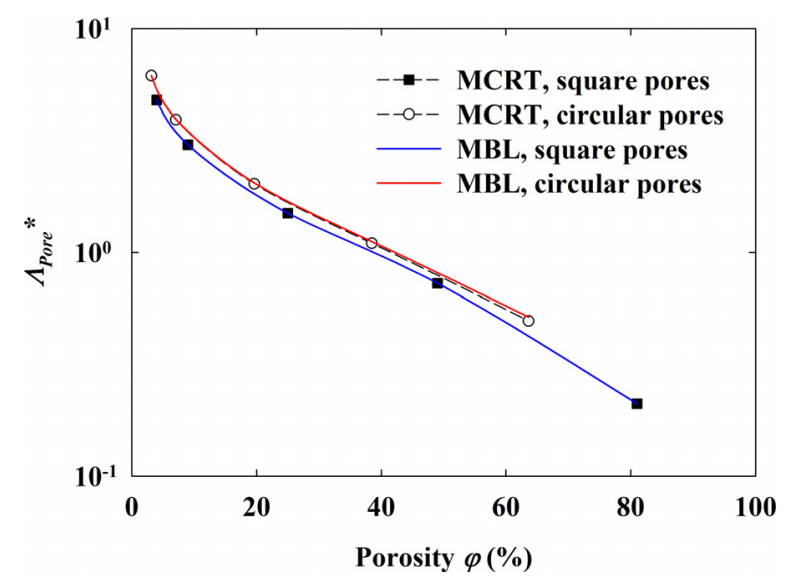

FIG. 2. Porosity-dependent $\Lambda_{\text {Pore }}^{*}$ given by the MCRT method and MBL.

principle, $\Lambda_{\text {Pore }}$ approaches zero at $\varphi \rightarrow 1$, but Eq. (7) predicts a nonzero $\Lambda_{\text {Pore }}$ value for $\varphi \approx 1$. Due to the error at high $\varphi$ values, this $\Lambda_{\text {Pore }}$ will not be used in the following analysis.

\section{Effective $\Lambda_{\text {Pore }}$ for periodic nanoporous thin films}

Although $\Lambda_{\text {Pore }}$ extracted in Section II B can be used in Eq. (2), the computed $k_{L}$ by Eq. (1) is often found to be less accurate. ${ }^{34}$ This divergence can be attributed to the usage of the Matthiessen's rule to add boundary scattering that is intrinsically different from the internal phonon scattering inside a material. ${ }^{22}$ The MCRT method can also be used to directly extract the modified phonon MFP $\Lambda_{i}(\omega)$ by further considering the internal phonon scattering. In this case, the shorter free traveling distance of phonons by internal and boundary scattering is selected and averaged to yield the reduced phonon MFPs. ${ }^{13}$ However, such $\Lambda_{i}(\omega)$ selection violates the Matthiessen's rule that assumes independent scattering mechanisms. ${ }^{50}$

To be consistent with the Matthiessen's rule, $\Lambda_{\text {Pore }}$ in Eq. (2) can be adjusted so that $k_{L}$ predicted by Eq. (1) can exactly match $k_{L}$ computed by frequency-dependent phonon MC simulations. This provides a simple way to extract the effective $\Lambda_{\text {Pore }}$. The phonon MC simulation adopts the recently proposed deviational phonon $\mathrm{MC}$ technique ${ }^{51}$ to improve the computational efficiency. As input parameters, an isotropic phonon dispersion is assumed and is taken from that for the (001) direction. The frequency-dependent bulk phonon MFPs are modified from that computed by first principles for the whole $k$ space. ${ }^{52}$ It is found that the reported scattering rates for the normal and Umklapp processes of transverse acoustic phonons should be both divided by 1.72 to fit the first-principles data, with all other parameters unchanged. The corresponding relaxation times of phonon-phonon scattering is given in Appendix B and more details can be found in our previous studies. ${ }^{53}$ Nanoporous Si thin films with aligned square and circular pores are computed as representative cases, with the period $p$ of $50-500 \mathrm{~nm}$ and the porosity $\varphi$ of roughly $4 \%-80 \%$. Within most of these simulated cases, the dimensionless $\Lambda_{\text {Pore }}^{*}$ changes within less than $5 \%$ for the same $\varphi$, as shown in Figs. 3(a) and 3(b). The averaged $\Lambda_{\text {Pore }}^{*}$ value is used in Eq. (2) and then Eq. (1) for $k_{L}$ predictions. In Figs. 4(a) and 4(b), the model predictions using $\Lambda_{\text {Pore }}^{*}$ (solid lines) agree well with 

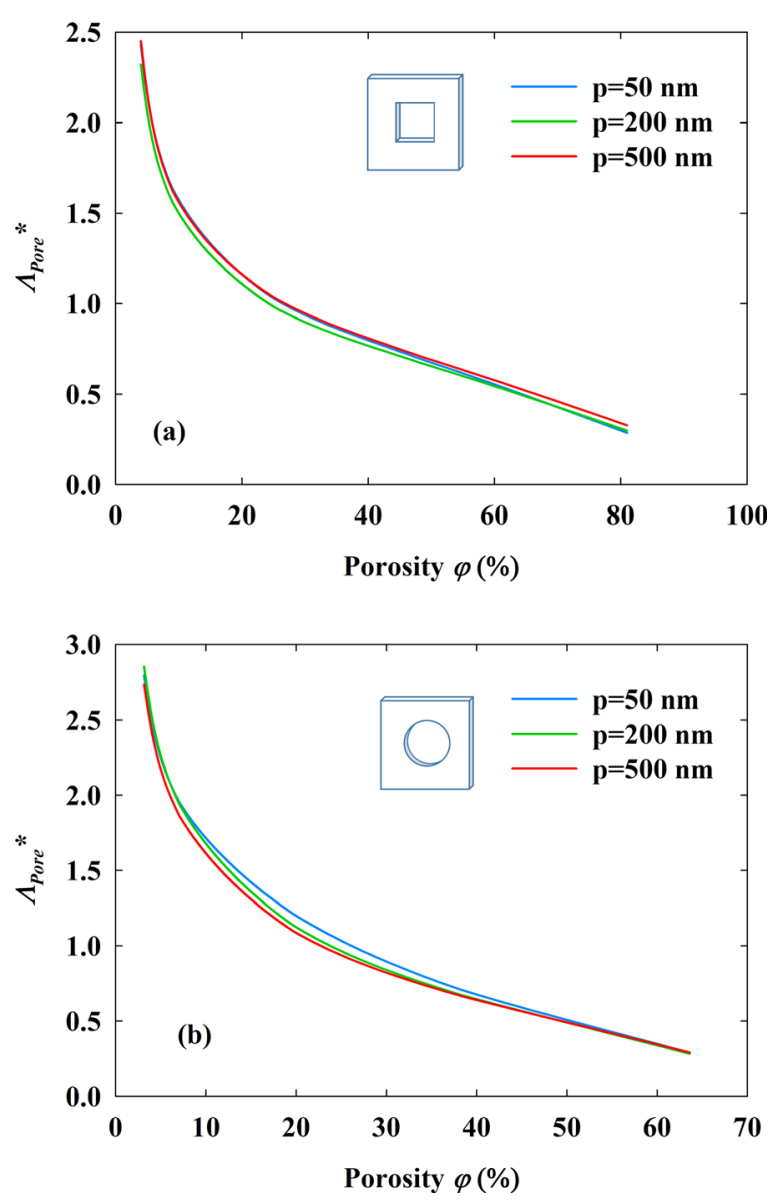

FIG. 3. Porosity- and period-dependent $\Lambda_{\text {Pore }}^{*}$ as extracted by matching the kinetic relationship in Eq. (1) with frequency-dependent phonon MC simulations on nanoporous Si thin films: (a) square pores and (b) circular pores.

frequency-dependent phonon MC simulations (symbols). The overall divergence is less than $5 \%$.

The averaged $\Lambda_{\text {Pore }}^{*}$ curve is further validated with Ge nanoporous thin films. Same as $\mathrm{Si}$, the (001) direction phonon dispersion is enforced for all directions. Again the scattering rates for the normal and Umklapp processes of the transverse acoustic phonons are reduced by a factor of 1.72 to exactly fit the first-principles calculations for the whole $k$ space $^{52}$ (see Appendix B). In Figs. 4(c) and 4(d), good agreement is found for Ge thin films with aligned square and cylindrical pores, respectively. For both $\mathrm{Si}$ and $\mathrm{Ge}$, their frequency-dependent phonon MFPs span from nanometers to micrometers. Similar phonon MFP spectra can also be found in many other materials. ${ }^{54,55}$ Therefore, the provided $\Lambda_{\text {Pore }}^{*}$ curve can be accurate for nanoporous thin films of a variety of materials, such as oxides and nitrides.

In comparison, all cases in Figs. 4(a)-4(d) are recomputed using $\Lambda_{\text {Pore }}$ by the MCRT method or the identical MBL. It is clear that $k_{L}$ is overestimated in this case. However, $\Lambda_{\text {Pore }}$ using the MBL can become accurate when extremely strong ballistic phonon transport exists, such as GALs with ultrafine porous structures and intrinsically long bulk phonon MFPs. More discussions will be given in Section II D.

In Figs. 5(a) and 5(b), the averaged $\Lambda_{\text {Pore }}^{*}$ is plotted as a function of the porosity $\varphi$ and dimensionless volumetric surface area $A^{*}$, respectively. The MCRT or MBL $\Lambda_{\text {Pore }}^{*}$ values are also plotted for comparison purpose. Here $A^{*}=p \cdot A$ and $A$ is the volumetric pore-surface area. In Fig. 5(a), it can be observed that $\Lambda_{\text {Pore }}^{*}$ values for different pore shapes start to deviate at $\varphi$ around 25\%. In Fig. 5(b), such divergence occurs above $A^{*}$ around 1.2. In both figures, the MCRT/ MBL results are generally above the extracted $\Lambda_{\text {Pore }}^{*}$, except for square pores with $\varphi>55 \%$.

\section{Effective $\Lambda_{\text {Pore }}$ for GALs with sub-100 $\mathrm{nm}$ porous structures}

The proposed strategy for phonon transport analysis can be easily extended to GALs with sub-100 nm structure sizes, as often encountered in experimental studies. ${ }^{56}$ As discussed earlier, phononic effects are not important for such structure sizes above $300 \mathrm{~K}$. As a simple case, internal phonon scattering can be neglected for sub- $100 \mathrm{~nm}$ neck width between adjacent pores because only $\sim 5 \%$ thermal conductivity is contributed by phonons with MFPs less than $70 \mathrm{~nm}$ in pristine graphene. ${ }^{57}$ It should be noted that the most reliable $k_{L}$ of graphene-based structures can be computed with $a b$ initio phonon studies. ${ }^{58-63}$ However, these studies are infeasible for the proposed structure size due to extremely high computational load. Similar to thin films, $k_{L}$ estimation is carried out here, assuming ballistic phonon transport within the GAL.

Because phonons only have two degrees of freedom in $2 \mathrm{D}$ graphene, $\Lambda_{\text {Pore }}^{*}$ is shorter than the values for nanoporous thin films. Figure 6(a) shows the extracted $\Lambda_{\text {Pore }}^{*}$ for circular pores. Following the idea of MBL,

$$
\Lambda_{\text {Pore }}=C \frac{p^{2}-\pi(d / 2)^{2}}{\pi d}
$$

is anticipated. By fitting the MCRT data, a constant $C$ value of 3.4 is found for circular pores.

For graphene, the detailed derivation of $k_{L}$ can be found in previous studies. ${ }^{64}$ Following this, Eq. (1) is changed to

$$
k_{L} \approx \frac{1}{2} F(\varphi) \Lambda_{\text {Pore }} \sum_{i, q} \int c_{i}(q) v_{g, i}(q) / t N \Omega,
$$

where $N$ is the number of wave vector $\mathbf{q}$ in the first Brillouin zone, $\Omega$ is area of a unit cell, and $t$ is the graphene thickness as $0.335 \mathrm{~nm}$. Here, the factor $1 / 3$ in Eq. (1) is changed to $1 / 2$ due to the reduced degree of freedom and $F(\varphi)$ is still taken from Fig. 1. In graphene, acoustic phonon branches include the out-of-plane, transverse, and longitudinal branches. Instead of simplified phonon dispersions, the exact phonon dispersion of pristine graphene is used in Eq. (9) and is computed with Vienna Ab-initio Simulation Package (VASP) ${ }^{65}$ and Phonopy package. ${ }^{66}$ Figure $6(\mathrm{~b})$ shows $H(T)=\sum_{i, q} \int c_{i}(\mathbf{q})$ $v_{g, i}(\mathbf{q}) / t N \Omega$ that can be used for quick thermal analysis when ballistic phonon transport and noncoherent phonons can be assumed within GALs.

For reduced structure sizes and/or temperatures, phononic effects may become critical and the modified phonon dispersion should be employed in the calculation. Similar treatment can be found for nanoporous Si thin films. ${ }^{14}$ In a more rigorous treatment, the characteristic length $\Lambda_{\text {Pore }}$ may also be changed due to partially specular and partially 

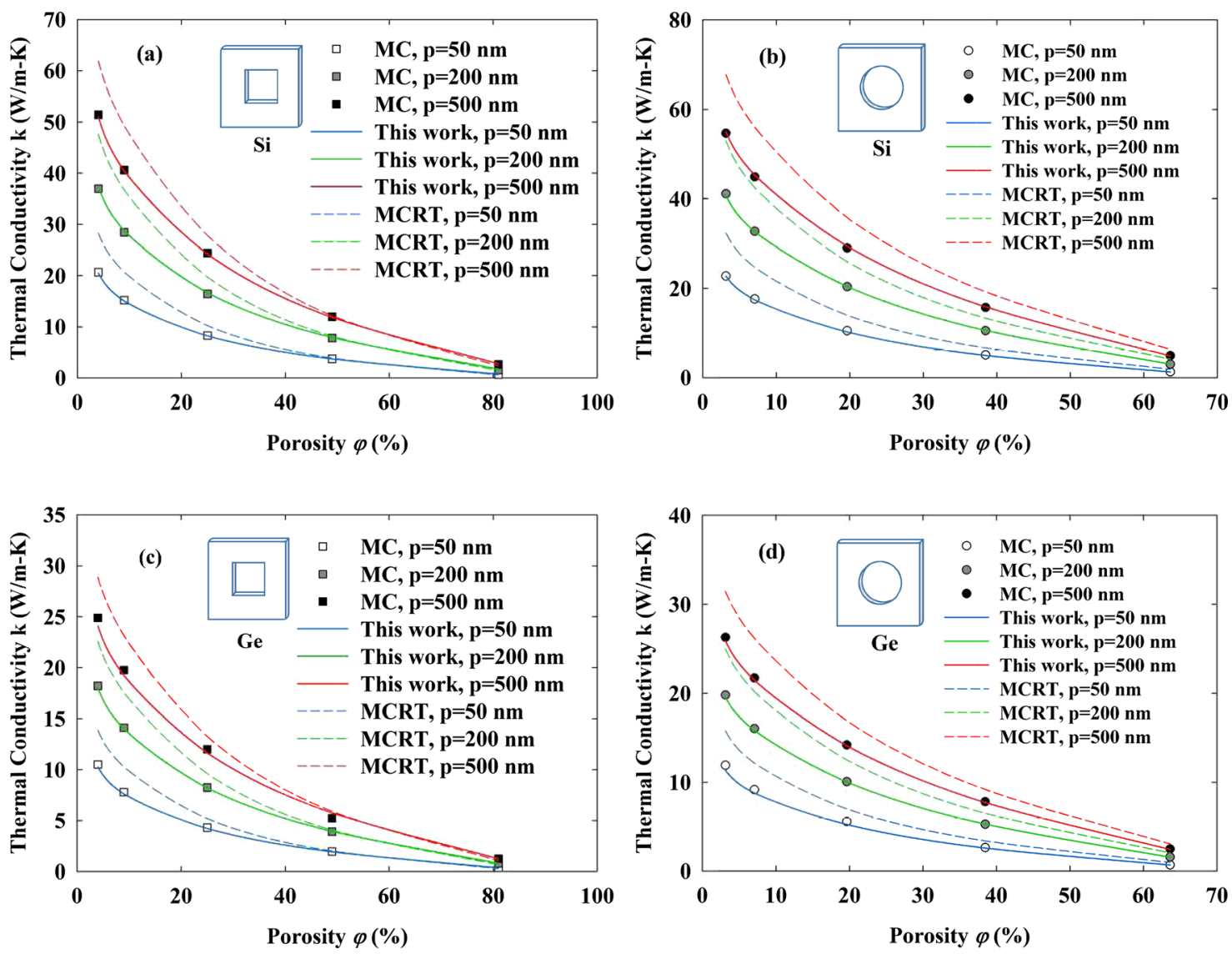

FIG. 4. Predicted $k_{L}$ of thin films with aligned pores, using $\Lambda_{\text {Pore }}^{*}$ averaged over the three curves in Figs. 3(a) and 3(b) (solid lines), and the MRCT/MBL method (dashed lines): (a) square pores in $\mathrm{Si}$, (b) circular pores in $\mathrm{Si}$, (c) square pores in $\mathrm{Ge}$, and (d) circular pores in Ge. The symbols are data from frequency-dependent phonon MC simulations.

diffusive pore-edge scattering of phonons. Specular phonon reflection by pore edges is required for phononic effects because diffusive phonon scattering by pore edges will destroy the phonon phase information and thus the phonon coherence, as suggested for the interfacial phonon scattering within superlattice thin films. ${ }^{67}$ More discussions are given in Section II E.

When defects exist, the internal phonon scattering should also be considered because the phonon MFPs can be reduced below the neck width between pores. In this case, Eq. (9) should be replaced by Eq. (1), except for the beginning $1 / 3$ factor replaced by $1 / 2$. The phonon relaxation times for different scattering mechanisms can be found in the literature. ${ }^{64}$ Based on previous analysis on nanoporous $\mathrm{Si}$ and Ge films, some overestimation is anticipated for $k_{L}$ based on MCRT/ MBL $\Lambda_{\text {Pore }}$ values. More accurate predictions can be obtained from phonon MC simulations using the ab initio three phonon scattering operator ${ }^{68}$ instead of the single-mode relaxation time approximation in the derivation Eq. (9). ${ }^{64}$ For graphene, it has been pointed out that the single-mode relaxation time approximation fails to reproduce the details of phononphonon scattering from ab initio modeling. ${ }^{58,61-63}$

\section{E. Partially specular and partially diffusive phonon scattering on pore edges}

This work mainly focuses on rough pore edges with completely diffusive phonon scattering. At cryogenic temperatures, however, specular pore-edge phonon scattering becomes important when pore-edge roughness becomes much smaller than the dominant phonon wavelength $\lambda_{d}$ as $1.48 \hbar v_{S} / k_{B} T{ }^{69}$ Here, $k_{B}$ is the Boltzmann constant, $T$ is the absolute temperature, $\hbar$ is Planck's constant divided by $2 \pi$, and $v_{S}$ is the sound velocity.

As an extreme case, completely specular phonon reflection on pore edges is assumed to recalculate the MCRT curves in Fig. 2 but the variation of $\Lambda_{\text {Pore }}$ is within $1 \%$ for both samples with circular and square pores. However, $\Lambda_{\text {Pore }}$ simply by the MCRT method does not consider the phonon momentum loss that introduces thermal resistance along the heat flow direction. To check this influence, the curves in Figs. 4(a) and 4(b) are recalculated in Figs. 7(a) and 7(b) with frequency-dependent phonon MC simulations, assuming atomically smooth pore edges and thus completely specular pore-edge phonon reflection. For both square and circular pores, smooth pore edges lead to higher $k_{L}$ values, particularly for square pores with two side walls parallel to the heat flow direction.

\section{ANALYSIS OF EXPERIMENTAL RESULTS}

The $k_{L}$ values predicted using extracted $\Lambda_{\text {Pore }}^{*}$ in Fig. 5(a) is compared with representative experimental data on Si thin films drilled by deep RIE ${ }^{2,8,15}$ or electrolytic etching $^{70}$ along the cross-plane direction. Here, electrolytic etching is anticipated to introduce more surface defects to further 

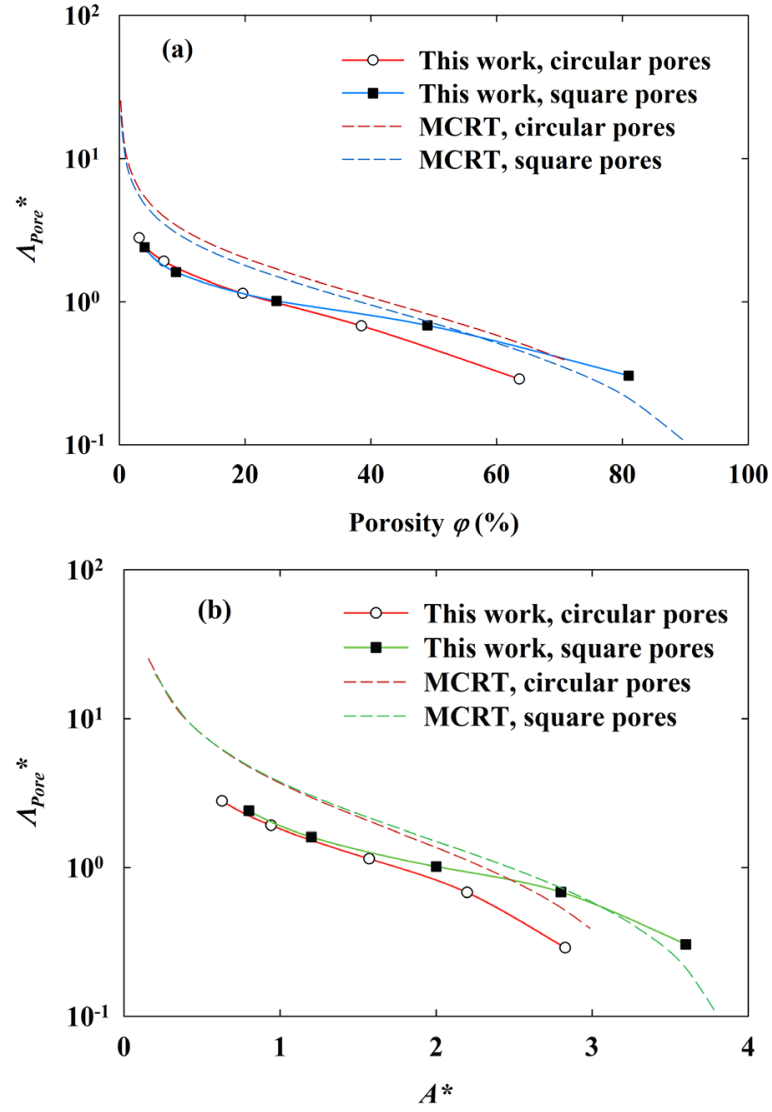

FIG. 5. Extracted $\Lambda_{\text {Pore }}^{*}$ (solid lines) and MCRT/MBL $\Lambda_{\text {Pore }}^{*}$ (dashed lines) as a function of: (a) porosity $\varphi$ and (b) dimensionless volumetric surface area $A^{*}$.

lower $k_{L}$, as suggested for rough silicon nanowires formed by electroless etching. ${ }^{71}$

Figure 8 compares the measured in-plane $k_{L}$ of nanoporous thin films (symbols) with the model predictions for corresponding periods (curves). Although the employed $\Lambda_{\text {Pore }}^{*}$ is extracted for nanoporous silicon films with $50-500 \mathrm{~nm}$ periods, the model prediction (red curve) agrees well with the data for a nanoporous Si thin film with $4 \mu \mathrm{m}$ period (red square). ${ }^{2}$ For a series of nanoporous Si thin films with $p=500-900 \mathrm{~nm}$ and $d=213-532 \mathrm{~nm},{ }^{15}$ the experimental data (green $\mathrm{X}$ ) are generally higher than the predicted upper and lower bounds (green curves). For nanoporous silicon films with hexagonally aligned pores and pitch of $55-350 \mathrm{~nm},{ }^{8}$ the corresponding period is computed as $48-296 \mathrm{~nm}$ for a square array based on the porosity $(\varphi=35 \%)$ and pore diameter. In general, the measurement data (purple circles) are lower than the predictions (purple lines).

Besides pores drilled by deep RIE, heavily doped silicon films with electrolytically etched pores ${ }^{70}$ are also modeled. For these samples, the electrical resistivity of $1-5 \mathrm{~m} \Omega \mathrm{cm}$ corresponds to boron doping range of $2.1 \times 10^{19}$ to 1.16 $\times 10^{20} \mathrm{~cm}^{-3}$. In these samples, phonons are also scattered by the inserted dopant atoms, their associated strain fields, and other unknown defects. The corresponding phonon scattering rate is given as $\tau_{I}^{-1}(\omega)=a \omega^{4} .{ }^{72}$ The constant $a$ combines the above three scattering mechanisms. For n-type single-crystal silicon films of comparable doping levels, $a$ ranges from $6.2 \times 10^{-45}$ to $1.46 \times 10^{-44} \mathrm{~s}^{3} .^{73}$ Without more accurate values, $a$ is set to be $1.0 \times 10^{-44} \mathrm{~s}^{3}$. For these films, pores have
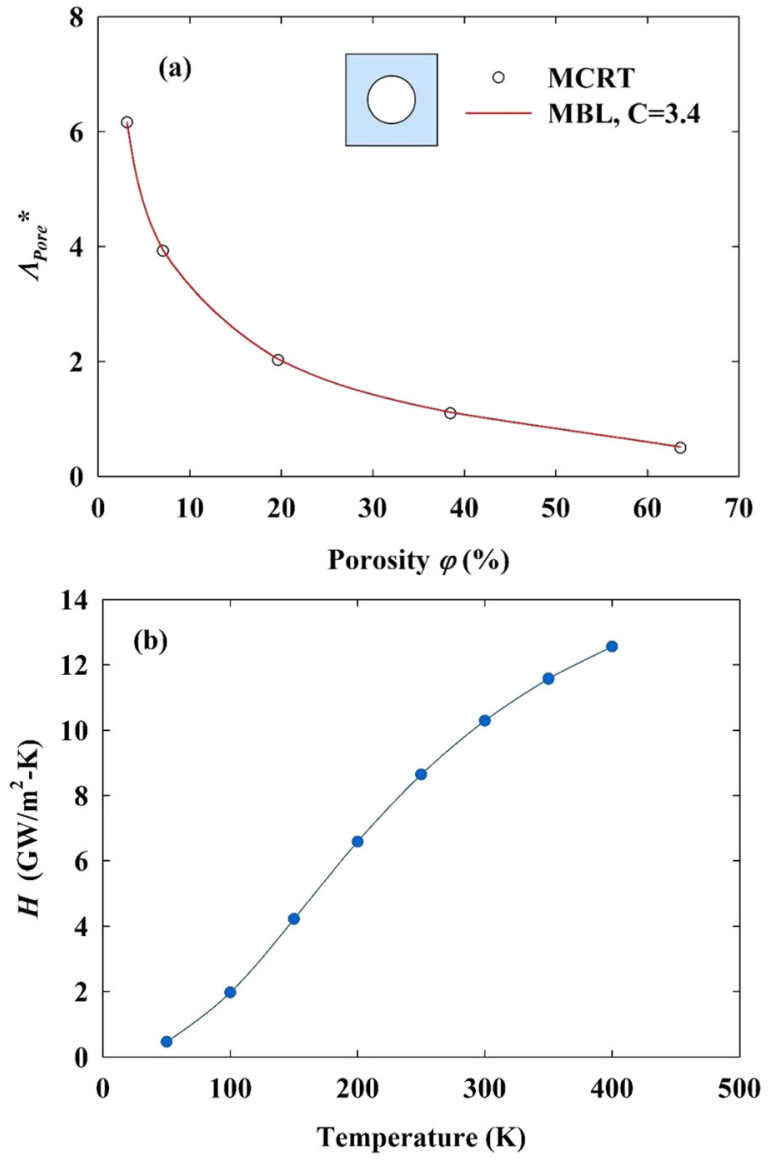

FIG. 6. (a) Extracted $\Lambda_{\text {Pore }}^{*}$ of GALs by the MCRT method (symbols) and the fitted MBL expression (line). (b) Computed $H(T)$ function for pristine graphene.

10-100 nm diameters, but the neck width between adjacent pores is constantly around $10 \mathrm{~nm}$. Based on the porosity and neck width, the averaged period $p$ is estimated as $78 \mathrm{~nm}$ (solid black curve). In general, the experimental data are lower than the predictions, which can be attributed to the increased poreedge defects introduced by wet etching. In molecular dynamic simulations on nanoporous thin films, the impact of amorphous pore edges has been emphasized. ${ }^{1}$ Similar effects have been suggested as the mechanism for the 100 -fold $k_{L}$ reduction of rough silicon nanowires. ${ }^{74}$ With $\sim 10 \mathrm{~nm}$ neck width as the characteristic length, phonon confinement may also lead to modified phonon dispersion, which will reduce the phonon group velocities and thus $k_{L}{ }^{75-77}$ More experimental studies may be carried out on films with varied feature sizes of the porous structure to elucidate these effects.

\section{SUMMARY}

In this work, the characteristic length $\Lambda_{\text {Pore }}$ for nanoporous thin films and GALs is extracted for phonon transport analysis. Despite the definition difference, $\Lambda_{\text {Pore }}$ acquired by the MCRT method is identical with the MBL that can be easily computed for a porous structure. The MCRT/MBL $\Lambda_{\text {Pore }}$ values are accurate with ballistic phonon transport. When phonon scattering within the material is still important, these $\Lambda_{\text {Pore }}$ values are generally higher than those extracted by matching the kinetic model with the prediction from phonon 

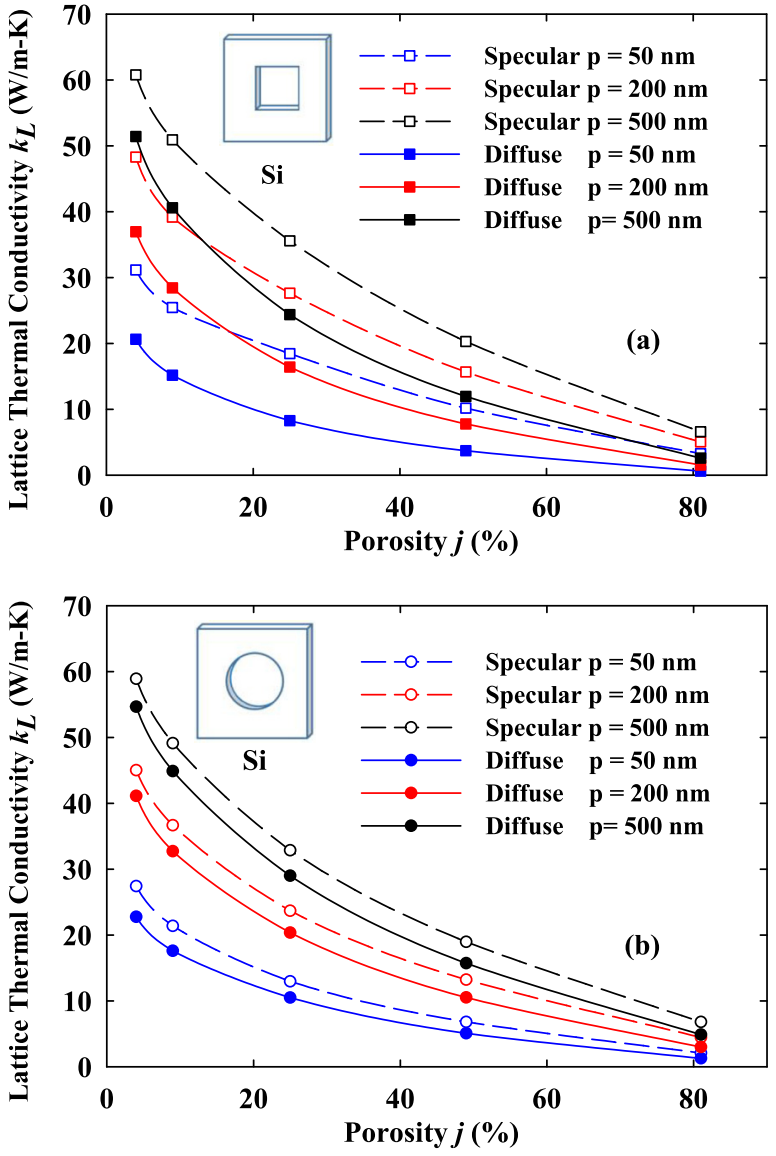

FIG. 7. Predicted $k_{L}$ of thin films with aligned (a) square pores and (b) circular pores. The dashed lines are for smooth pore edges with complete specular phonon reflection, whereas the solid lines are for rough pore edges with complete diffusive phonon reflection.

MC simulations. It is found that the dimensionless $\Lambda_{\text {Pore }}^{*}$ can be applied to these nanoporous structures up to a certain porosity or dimensionless volumetric pore-surface area, regardless of the pore shape and porous structure size. In addition, the widely used Eucken and Russell factors for porosity influence is also corrected by Fourier analysis for 2D porous structures and large divergence has been found at high porosities. Using the extracted $\Lambda_{\text {Pore }}^{*}$, the predicted $k_{L}$ is in good agreement with the frequency-dependent phonon MC simulations for both nanoporous $\mathrm{Si}$ and $\mathrm{Ge}$ thin films. The

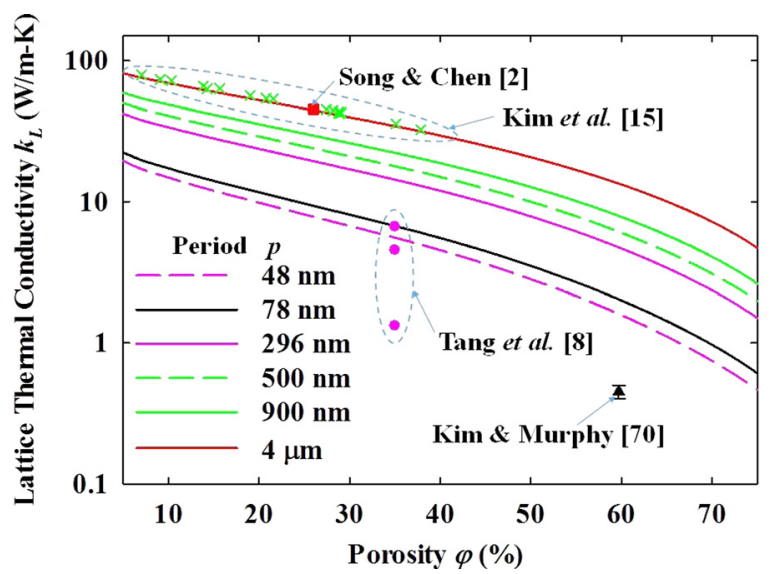

FIG. 8. Comparison between experimental results and the model predictions. predictions also match well with some of the existing experimental results. In contrast, $\Lambda_{\text {Pore }}^{*}$ given by MCRT or MBL often leads to overestimated $k_{L}$, especially at high porosities.

The developed analytical model provides a simple approach for $k_{L}$ calculations. The time-consuming phonon MC simulations can thus be replaced with the new model for both data analysis and materials design. Beyond phonon studies, the extracted $\Lambda_{\text {Pore }}^{*}$ can also be used for electron modeling, in which the bulk electron MFPs can be modified to predicted the electrical conductivity and Seebeck coefficient.

\section{ACKNOWLEDGMENTS}

The authors thank Dr. Jean-Philippe M. Péraud for the help of deviational phonon MC simulations and Professor David Broido for discussions on bulk phonon MFPs for $\mathrm{Si}$ and Ge. We acknowledge the support from the U.S. Air Force Office of Scientific Research (Award No. FA9550-161-0025). An allocation of computer time from the UA Research Computing High Performance Computing (HPC) and High Throughput Computing (HTC) at the University of Arizona is gratefully acknowledged.

\section{APPENDIX A: TREATMENT OF POROUS THIN FILMS OR 2D MATERIALS IN PHONON MC SIMULATIONS USING RECTANGULAR SUBCELLS}

The computational domain, which is divided into identical rectangular subcells, has to be modified to accommodate the cylindrical pores. For subcells intercepting with the pore edge, only the volume outside the pore will be used in the simulation. This partial volume is evaluated by the MC technique. First, at least half a million particles are randomly distributed within each of these subcells. The percentage of the subcell volume outside the pore is set as the percentage of particles outside the sphere. This partial volume is employed in counting the phonon energy density of each subcell, which is used to update the subcell temperature during the simulation. ${ }^{10}$

For porous thin films, diffusive phonon reflection at point A (Fig. 9 as the $x-y$ plane projection) gives the new traveling direction as

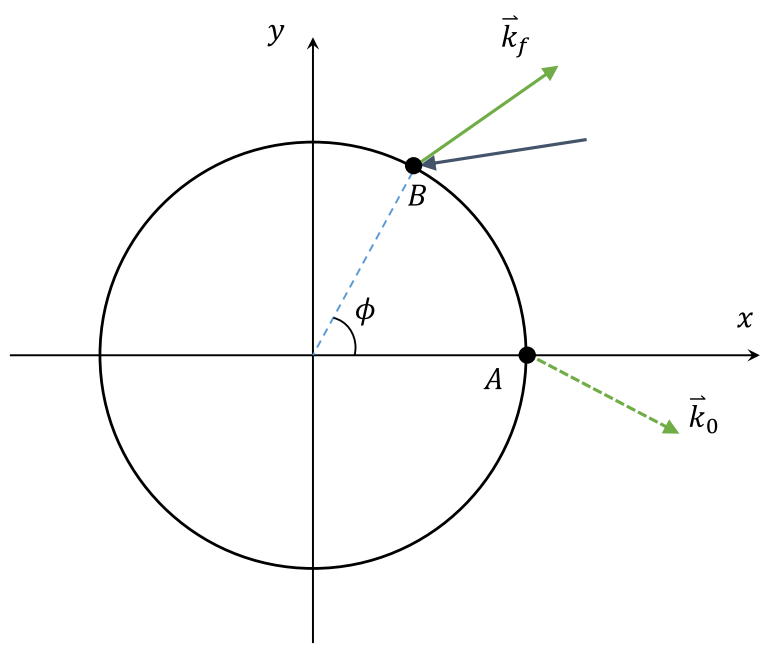

FIG. 9. Traveling direction reset of phonons diffusively reflected by a pore edge. 
TABLE I. Parameters used for phonon-phonon scattering rates of bulk Si and Ge. In the given relaxation time expressions, $\hbar \omega$ should be converted into meV to be consistent with the unit of $A_{p, N / U}$ values.

\begin{tabular}{|c|c|c|c|c|c|c|c|}
\hline Material & $A_{T A, N}\left(\mathrm{meV}^{2} \mathrm{~K} \mathrm{~s}\right)^{-1}$ & $A_{L A, N}\left(\mathrm{meV}^{2} \mathrm{~K} \mathrm{~s}\right)^{-1}$ & $A_{T A, U}\left(\mathrm{meV}^{4} \mathrm{~K} \mathrm{~s}\right)^{-1}$ & $A_{L A, U}\left(\mathrm{meV}^{4} \mathrm{~K} \mathrm{~s}\right)^{-1}$ & $\Theta_{D}(\mathrm{~K})$ & $g$ & $V_{0}\left(\mathrm{~m}^{3}\right)$ \\
\hline $\mathrm{Si}$ & 147280 & 163921 & 1170 & 507 & 645 & $2.01 \times 10^{-4}$ & $2.0 \times 10^{-29}$ \\
\hline $\mathrm{Ge}$ & 267586 & 401980 & 5020 & 1990 & 374 & $5.89 \times 10^{-4}$ & $2.26 \times 10^{-29}$ \\
\hline
\end{tabular}

$$
\vec{k}_{0}=\left[\begin{array}{c}
k_{x} \\
k_{y} \\
k_{z}
\end{array}\right]=\left[\begin{array}{c}
\sqrt{R_{1}} \\
\left(\sqrt{1-R_{1}}\right) \cos \left(2 \pi R_{2}\right) \\
\left(\sqrt{1-R_{1}}\right) \sin \left(2 \pi R_{2}\right)
\end{array}\right],
$$

where $R_{1}$ and $R_{2}$ are two random numbers between 0 and 1 . For phonon scattered at point $\mathrm{B}$ on the pore edge, the outbound $\vec{k}_{f}$ can be obtained by rotating $\vec{k}_{0}$ from point A by an angle $\phi$ to point $\mathrm{B}$ within the same $\mathrm{x}$-y plane. As a result, $\vec{k}_{f}$ is described as

$$
\vec{k}_{f}=\left[\begin{array}{c}
k_{x} \cos \phi-k_{y} \sin \phi \\
k_{y} \cos \phi+k_{x} \sin \phi \\
k_{z}
\end{array}\right] .
$$

For 2D porous materials such as GALs, $k_{z}$ is fixed at zero and $R_{2}$ in Eq. (A1) is randomly set as 0 or 1 . The same procedure can then be used to find $k_{f}$.

\section{APPENDIX B: PHONON SCATTERING RATES OF BULK SI AND GE}

In all simulations, an isotropic phonon dispersion is assumed and is taken along the (001) direction for bulk silicon and germanium. The overall phonon relaxation time $\tau_{i}(\omega)$ for branch $i$, defined as $\Lambda_{B u l k, i}(\omega) / v_{g, i}(\omega)$, is computed based on the Matthiessen's rule

$$
\tau_{i}^{-1}(\omega)=\tau_{I}^{-1}(\omega)+\tau_{i, N}^{-1}(\omega)+\tau_{i, U}^{-1}(\omega),
$$

where $\tau_{I}(\omega), \tau_{i, N}(\omega)$, and $\tau_{i, U}(\omega)$ are the relaxation times of impurity scattering, normal process, and Umklapp process, respectively. All utilized parameters are summarized in Table I for reference. The detailed expressions of these relaxation times are given below.

For undoped samples, $\tau_{I}(\omega)$ is only due to isotope atoms and is given as ${ }^{78}$

$$
\tau_{I}^{-1}(\omega)=\frac{\pi}{6} V_{0} g \omega^{2} \sum_{i} D_{i}(\omega),
$$

where $V_{0}$ is the averaged volume per atom, $g$ is the mass variance determined by the composition, and $D_{i}(\omega)$ is the phonon density of states for branch $i$. In the low-frequency limit $(\omega \rightarrow 0)$, Eq. (B2) will degrade to the widely used formulation, $\tau_{I}^{-1}(\omega)=a \omega^{4}{ }^{72}$

Based on first-principles calculations on bulk $\mathrm{Si}$ and $\mathrm{Ge}$, the relaxation times of the momentum-conserved normal process and momentum-non-conserved Umklapp process are calculated as ${ }^{52}$

$$
\tau_{p, N}^{-1}(\omega)=A_{p, N}(\hbar \omega)^{2} T\left[1-\exp \left(-3 T / \Theta_{D}\right)\right],
$$

$$
\tau_{p, U}^{-1}(\omega)=A_{p, U}(\hbar \omega)^{4} T\left[1-\exp \left(-3 T / \Theta_{D}\right)\right],
$$

in which $\hbar$ is the Planck's constant divided by $2 \pi, T$ is the absolute temperature, $\Theta_{D}$ is the Debye temperature, and $A_{p, N / U}$ is material-dependent coefficient. With Eq. (1), $A_{p, N / U}$ values are determined by fitting the temperature-dependent $k_{L}$ values for the LA and TA branches, both extracted from the same first-principles calculations. ${ }^{79}$ Because the phonon dispersions are enforced to be isotropic here, it is found that the reported $A_{T A, N}$ and $A_{T A, U}$ values need to be divided by 1.72 to fit the first-principles $k_{L, T A}$ data computed for the whole $k$ space, whereas $A_{L A, N}$ and $A_{L A, U}$ values are unaffected.

${ }^{1}$ Y. He, D. Donadio, J.-H. Lee, J. C. Grossman, and G. Galli, ACS Nano 5, 1839 (2011).

${ }^{2}$ D. Song and G. Chen, Appl. Phys. Lett. 84, 687 (2004).

${ }^{3}$ P. E. Hopkins, C. M. Reinke, M. F. Su, R. H. Olsson, E. A. Shaner, Z. C. Leseman, J. R. Serrano, L. M. Phinney, and I. El-Kady, Nano Lett. 11, 107 (2011).

${ }^{4}$ J.-K. Yu, S. Mitrovic, D. Tham, J. Varghese, and J. R. Heath, Nat. Nanotechnol. 5, 718 (2010).

${ }^{5}$ J. H. Lee, J. C. Grossman, J. Reed, and G. Galli, Appl. Phys. Lett. 91, 223110 (2007).

${ }^{6}$ G. Romano, A. Di Carlo, and J. C. Grossman, J. Comput. Electron. 11, 8 (2012).

${ }^{7}$ G. Romano and J. C. Grossman, Appl. Phys. Lett. 105, 033116 (2014).

${ }^{8}$ J. Tang, H.-T. Wang, D. H. Lee, M. Fardy, Z. Huo, T. P. Russell, and P. Yang, Nano Lett. 10, 4279 (2010).

${ }^{9}$ J. Lee, J. Lim, and P. Yang, Nano Lett. 15, 3273 (2015).

${ }^{10}$ Q. Hao, G. Chen, and M.-S. Jeng, J. Appl. Phys. 106, 114321 (2009).

${ }^{11} \mathrm{Q}$. Hao and G. Chen, in Applications of Monte Carlo Method in Science and Engineering, edited by S. Mordechai (InTech, 2011).

${ }^{12} \mathrm{Q}$. Hao, D. Xu, and H. Zhao, Systematic Studies of Periodically Nanoporous Si Films for Thermoelectric Applications (Mater. Res. Soc. Symp. Proc., San Francisco, California, 2015), p. mrss15.

${ }^{13}$ A. M. Marconnet, T. Kodama, M. Asheghi, and K. E. Goodson, Nanoscale Microscale Thermophys. Eng. 16, 199 (2012).

${ }^{14}$ E. Dechaumphai and R. Chen, J. Appl. Phys. 111, 073508 (2012).

${ }^{15}$ B. Kim, J. Nguyen, P. J. Clews, C. M. Reinke, D. Goettler, Z. C. Leseman, I. El-Kady, and R. Olsson, "Thermal conductivity manipulation in single crystal silicon via lithographycally defined phononic crystals," in IEEE 25th International Conference on Micro Electro Mechanical Systems (MEMS), 2012, p. 176

${ }^{16}$ S. Alaie, D. F. Goettler, M. Su, Z. C. Leseman, C. M. Reinke, and I. ElKady, Nat. Commun. 6, 7228 (2015).

${ }^{17}$ N. Zen, T. A. Puurtinen, T. J. Isotalo, S. Chaudhuri, and I. J. Maasilta, Nat. Commun. 5, 3435 (2014)

${ }^{18}$ P. Granitzer and K. Rumpf, Materials 3, 943 (2010).

${ }^{19}$ M. Maldovan, Phys. Rev. Lett. 110, 025902 (2013).

${ }^{20}$ A. Nassiopoulou and G. Kaltsas, Phys. Status Solidi A 182, 307 (2000).

${ }^{21}$ E. Hourdakis and A. G. Nassiopoulou, Sensors 13, 13596 (2013).

${ }^{22} \mathrm{G}$. Chen, Nanoscale Energy Transport and Conversion: A Parallel Treatment of Electrons, Molecules, Phonons, and Photons (Oxford University Press, New York, 2005).

${ }^{23}$ T. G. Pedersen, C. Flindt, J. Pedersen, N. A. Mortensen, A.-P. Jauho, and K. Pedersen, Phys. Rev. Lett. 100, 136804 (2008).

${ }^{24}$ Y. Yan, Q.-F. Liang, H. Zhao, C.-Q. Wu, and B. Li, Phys. Lett. A 376, 2425 (2012).

${ }^{25}$ D. G. Cahill, P. V. Braun, G. Chen, D. R. Clarke, S. Fan, K. E. Goodson, P. Keblinski, W. P. King, G. D. Mahan, A. Majumdar, H. J. Maris, S. R. Phillpot, E. Pop, and L. Shi, Appl. Phys. Rev. 1, 011305 (2014). 
${ }^{26}$ P. Hyldgaard and G. Mahan, Phys. Rev. B 56, 10754 (1997).

${ }^{27}$ M. Simkin and G. Mahan, Phys. Rev. Lett. 84, 927 (2000).

${ }^{28}$ S.-I. Tamura, Y. Tanaka, and H. J. Maris, Phys. Rev. B 60, 2627 (1999).

${ }^{29}$ J. Ravichandran, A. K. Yadav, R. Cheaito, P. B. Rossen, A. Soukiassian, S. J. Suresha, J. C. Duda, B. M. Foley, C.-H. Lee, Y. Zhu, A. W. Lichtenberger, J. E. Moore, D. A. Muller, D. G. Schlom, P. E. Hopkins, A. Majumdar, R. Ramesh, and M. A. Zurbuchen, Nat. Mater. 13, 168 (2014).

${ }^{30}$ J. Garg and G. Chen, Phys. Rev. B 87, 140302 (2013).

${ }^{31}$ A. Jain, Y.-J. Yu, and A. J. H. McGaughey, Phys. Rev. B 87, 195301 (2013).

${ }^{32}$ N. K. Ravichandran and A. J. Minnich, Phys. Rev. B 89, 205432 (2014).

${ }^{33}$ Z. Tian, K. Esfarjani, J. Shiomi, A. S. Henry, and G. Chen, Appl. Phys. Lett. 99, 053122 (2011).

${ }^{34}$ V. Jean, S. Fumeron, K. Termentzidis, S. Tutashkonko, and D. Lacroix, J. Appl. Phys. 115, 024304 (2014).

${ }^{35}$ J. Randrianalisoa and D. Baillis, J. Appl. Phys. 103, 053502 (2008).

${ }^{36}$ G. Romano and J. C. Grossman, J. Heat Transfer 137, 071302 (2015).

${ }^{37}$ J. D. Chung and M. Kaviany, Int. J. Heat Mass Transfer 43, 521 (2000).

${ }^{38}$ R. Prasher, J. Appl. Phys. 100, 034307 (2006).

${ }^{39}$ R. Prasher, J. Appl. Phys. 100, 064302 (2006).

${ }^{40}$ M. Asheghi, Y. K. Leung, S. S. Wong, and K. E. Goodson, Appl. Phys. Lett. 71, 1798 (1997).

${ }^{41}$ M. Asheghi, M. N. Touzelbaev, K. E. Goodson, Y. K. Leung, and S. S. Wong, J. Heat Transfer 120, 30 (1998).

${ }^{42}$ Y. S. Ju and K. E. Goodson, Appl. Phys. Lett. 74, 3005 (1999).

${ }^{43}$ E. Chávez-Ángel, J. S. Reparaz, J. Gomis-Bresco, M. R. Wagner, J. Cuffe, B. Graczykowski, A. Shchepetov, H. Jiang, M. Prunnila, J. Ahopelto, F. Alzina, and C. M. Sotomayor Torres, APL Mater. 2, 012113 (2014).

${ }^{44}$ A. Eucken, Ceram. Abstr. 11, 576 (1932).

${ }^{45}$ A. Eucken, Ceram. Abstr. 12, 231 (1933).

${ }^{46}$ H. Russell, J. Am. Ceram. Soc. 18, 1 (1935).

${ }^{47}$ D. Lacroix, K. Joulain, and D. Lemonnier, Phys. Rev. B 72, 064305 (2005).

${ }^{48}$ J. R. Howell, M. P. Menguc, and R. Siegel, Thermal Radiation Heat Transfer (CRC Press, 2010).

${ }^{49}$ A. Minnich and G. Chen, Appl. Phys. Lett. 91, 073105 (2007).

${ }^{50}$ A. J. H. McGaughey and A. Jain, Appl. Phys. Lett. 100, 061911 (2012).

${ }^{51}$ J.-P. M. Péraud and N. G. Hadjiconstantinou, Phys. Rev. B 84, 205331 (2011).

${ }^{52}$ A. Ward and D. A. Broido, Phys. Rev. B 81, 085205 (2010).
${ }^{53}$ Q. Hao, J. Appl. Phys. 116, 034305 (2014).

${ }^{54}$ J. P. Freedman, J. H. Leach, E. A. Preble, Z. Sitar, R. F. Davis, and J. A. Malen, Sci. Rep. 3, 2963 (2013).

${ }^{55}$ T. Feng and X. Ruan, J. Nanomater. 2014, 206370.

${ }^{56}$ A. Sandner, T. Preis, C. Schell, P. Giudici, K. Watanabe, T. Taniguchi, D. Weiss, and J. Eroms, Nano Lett. 15, 8402 (2015).

${ }^{57}$ T. Feng, X. Ruan, Z. Ye, and B. Cao, Phys. Rev. B 91, 224301 (2015).

${ }^{58}$ L. Lindsay, D. A. Broido, and N. Mingo, Phys. Rev. B 82, 115427 (2010).

${ }^{59}$ S. Lee, D. Broido, K. Esfarjani, and G. Chen, Nat. Commun. 6, 6290 (2015).

${ }^{60}$ N. Bonini, J. Garg, and N. Marzari, Nano Lett. 12, 2673 (2012).

${ }^{61}$ J. H. Seol, I. Jo, A. L. Moore, L. Lindsay, Z. H. Aitken, M. T. Pettes, X. Li, Z. Yao, R. Huang, D. Broido, N. Mingo, R. S. Ruoff, and L. Shi, Science 328, 213 (2010).

${ }^{62}$ D. Singh, J. Y. Murthy, and T. S. Fisher, J. Appl. Phys. 110, 094312 (2011).

${ }^{63}$ D. Singh, J. Y. Murthy, and T. S. Fisher, J. Appl. Phys. 110, 113510 (2011).

${ }^{64}$ D. L. Nika, E. P. Pokatilov, A. S. Askerov, and A. A. Balandin, Phys. Rev. B 79, 155413 (2009).

${ }^{65}$ J. Hafner, J. Comput. Chem. 29, 2044 (2008).

${ }^{66}$ A. Togo and I. Tanaka, Scr. Mater. 108, 1 (2015).

${ }^{67}$ B. Yang and G. Chen, Phys. Rev. B 67, 195311 (2003).

${ }^{68}$ C. D. Landon and N. G. Hadjiconstantinou, J. Appl. Phys. 116, 163502 (2014).

${ }^{69}$ T. Klitsner and R. O. Pohl, Phys. Rev. B 36, 6551 (1987).

${ }^{70}$ K. Kim and T. E. Murphy, J. Appl. Phys. 118, 154304 (2015).

${ }^{71}$ A. I. Hochbaum, R. Chen, R. D. Delgado, W. Liang, E. C. Garnett, M. Najarian, A. Majumdar, and P. Yang, Nature 451, 163 (2008).

${ }^{72}$ J. M. Ziman, Electrons and Phonons: The Theory of Transport Phenomena in Solids (Oxford University Press, 2001).

${ }^{73}$ M. Asheghi, K. Kurabayashi, R. Kasnavi, and K. Goodson, J. Appl. Phys. 91, 5079 (2002).

${ }^{74}$ Y. He and G. Galli, Phys. Rev. Lett. 108, 215901 (2012).

${ }^{75}$ A. Balandin and K. L. Wang, Phys. Rev. B 58, 1544 (1998).

${ }^{76}$ N. Bannov, V. Aristov, V. Mitin, and M. A. Stroscio, Phys. Rev. B 51, 9930 (1995).

${ }^{77}$ D. Li, Y. Wu, P. Kim, L. Shi, P. Yang, and A. Majumdar, Appl. Phys. Lett. 83, 2934 (2003).

${ }^{78}$ S.-I. Tamura, Phys. Rev. B 27, 858 (1983).

${ }^{79}$ A. Ward, Ph.D. thesis, Boston College, 2009. 